\title{
Nondestructive Evaluation of Foam Insulation for the External Tank Return to Flight
}

\author{
James L. Walker* and Joel D. Richter* \\ NASA/Marshall Space Flight Center, Huntsville, AL, 35812
}

\begin{abstract}
Nondestructive evaluation methods have been developed to identify defects in the foam thermal protection system (TPS) of the Space Shuttle External Tank (ET). Terahertz imaging and backscatter radiography have been brought from prototype lab systems to production hardened inspection tools in just a few years. These methods have been demonstrated to be capable of detecting void type defects under many inches of foam which, if not repaired, could lead to detrimental foam loss. The evolution of these methods from lab tools to implementation on the ET will be discussed.
\end{abstract}

\section{Introduction}

Goam loss from the External Tank (ET) of the Space Shuttle impacted the Orbiter Columbia's wing leading edge during the launch of STS-107 on January 16, 2003. The damage from this impact led to the breakup of the Orbiter on reentry 16 days later ${ }^{1}$. As a result of the accident, the National Aeronautics and Space Administration (NASA) began investigating in detail the mechanisms of Spray-On-Foam-Insulation (SOFI) loss and ways to mitigate it. As part of the mitigation effort, Nondestructive Evaluation (NDE) techniques that could identify defects in foam were investigated, developed and brought to bear on the subsequent "Return-to-Flight" (RTF) ET and backup ET's.

\section{Defect Types of Interest}

A prerequisite to inspecting foam, or any material, is an understanding of the nature of the critical defects which cause unacceptable performance of the material. Prior to the Columbia accident, mitigation of foam loss was primarily focused on "popcorning" caused by cohesive failures close to the surface of the foam and large delaminations caused by a process escape during spraying. After the accident, voids within the foam became the primary defect of concern and received extensive focus during the RTF period leading up to STS-114, launched on July 26,2005 . Testing was performed to correlate void size, shape and depth to foam debris mass loss under flightlike environmental profiles. Based on early testing, a basic understanding of the fracture properties of the material was formed and a basic critical defect criterion was established. From this work a 1.0" cylindrical void with a height of 0.5 " was initially determined to be the target size for any NDE method to find.

\section{External Tank Thermal Protection System (TPS) Overview}

The ET is comprised of three major structures which are, from top to bottom, the liquid oxygen tank, unpressurized intertank and the liquid hydrogen tank. The foam sprayed over the acreage of the tank is robotically applied to a thickness of about an inch. The loss of acreage foam is not a great concern to the Orbiter due to a good understanding and control of the application process, making it unlikely to come off and in the event that it did, not likely to be large because it is so thin. The areas of primary concern are regions of foam covering protuberances and final closeouts because that foam is applied by a less controllable hand spray process and are often thicker than the acreage. The hand spray process is required due to the complex substrate geometry often associated with the closeouts or protuberances and lends itself to a higher number of defects. The principle hand sprayed closeouts of concern from a debris generating point of view are the Ice Frost Ramps (IFR's), the flange joints between the tanks, the Protuberance Air Load (PAL) ramps and the Bipod close-outs. The thickest pieces of foam, and thus an area of particular concern, are the IFR's, which extend almost a foot above the aluminum tank skin. These areas have lost some foam over the course of the Shuttle Program but for the most part the mass of foam loss has been small. The flange between the intertank and the hydrogen tank is another area of special interest because it has historically been

\footnotetext{
" Materials Engineer, Damage Tolerance Assessment Branch, EM 20
} 
a high debris generation region. To a lesser degree the flange between the intertank and the oxygen tank is also of concern. The bipod ramps, although redesigned to be much smaller than the ones that flew on Columbia's final mission, are still thicker than the surrounding acreage foam and an area of continued interest. The PAL ramps which help to shield the cable tray and pressurization lines are very thick, large pieces of foam that received a lot of attention during the RTF period because of their size. These ramps were eliminated after the Discovery, STS114, flight. Figure 1 shows a picture of a labeled ET following separation from the Orbiter.

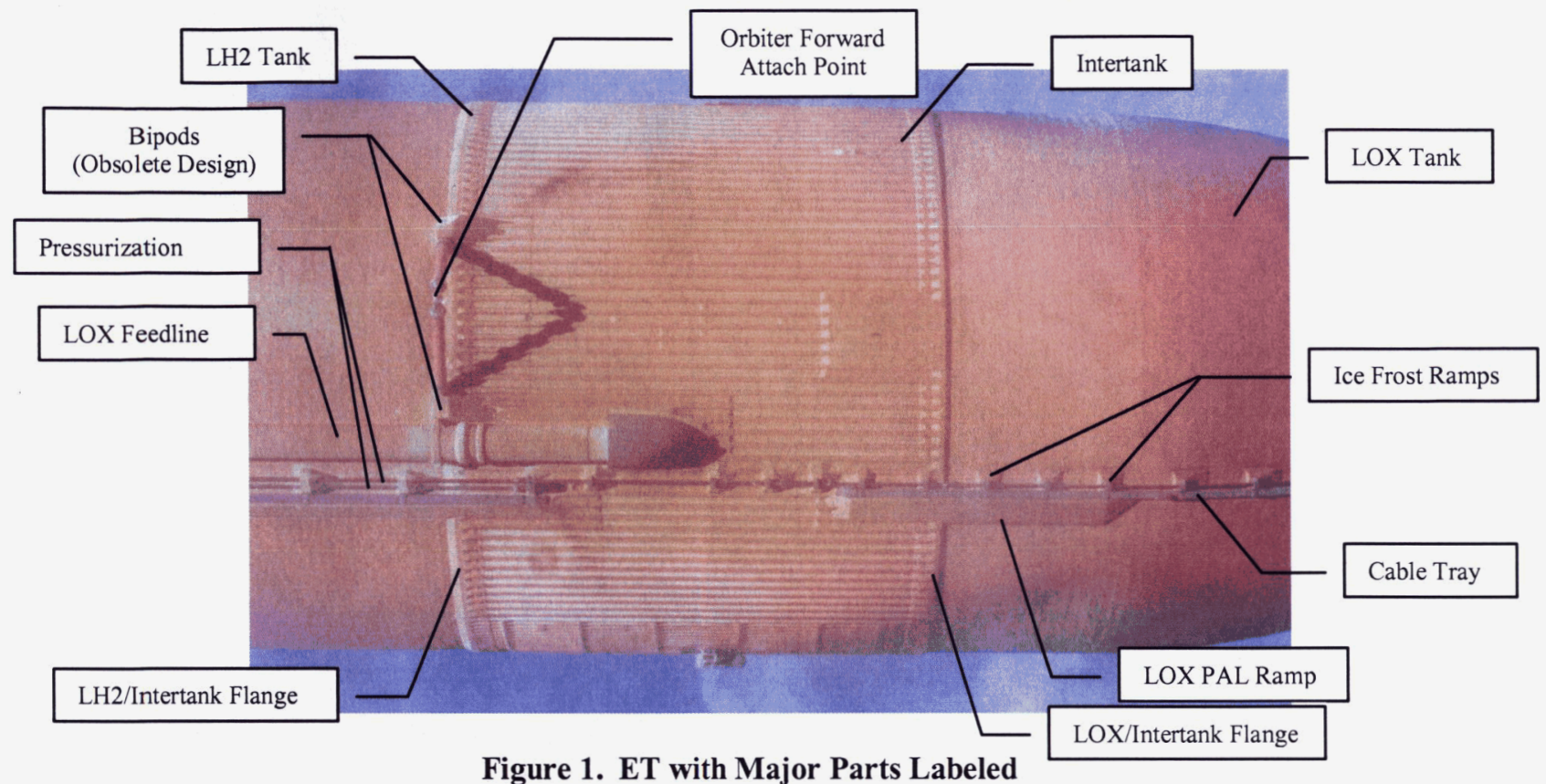

\section{Initial Candidate Technologies}

Prior to the Columbia accident there were no NDE methods being used to screen the ET SOFI for internal defects as the foam was considered to be non-structural and therefore did not require a fracture mechanics analysis. The only NDE that was performed on the SOFI of the finished tank was a visual inspection for damage. After the Columbia accident a search was made for candidate inspection technologies that could detect a void within the foam at depths of up to eight inches. The results of an industry wide search yielded well over a dozen vendors representing about ten different inspection methods. Screening tests with simple test panels were used to narrow the list of potential candidates to five which then participated in a down select competition. The five technologies were: Laser Doppler Vibrometry, Laser Shearography, Backscatter radiography, Continuous Wave Microwave and Pulsed Terahertz imaging. Each technology was represented by a different vendor or institution. After a brief period of development work, test panels with defects unknown to the vendors were inspected by each vendor in order to generate the data necessary to decide what to use to inspect SOFI on the ET.

The Laser Doppler Vibrometry technique relied on an acoustic source "spark" to excite the test article while a laser, scanned across the part, measured the surface displacement at each single point. The result is a map of the relative foam stiffness. The Laser Shearography technique employed used a similar measurement principle but used a laser fringe pattern to measure surface displacements over the entire field of view of a digital camera, allowing for more rapid inspections. Here, both an acoustic air horn and vacuum differential were used as the excitation sources. Continuous wave Microwave ${ }^{2}$ relied on a waveguide or lens to direct microwaves through a test article, reflect off the material and substrate then be received and measured near or at the source. The dielectric properties of the material and any discontinuities in the material cause the amplitude and phase of the wave to be altered relative to each other yielding a through thickness map of the structure.

Backscatter Radiography ${ }^{3}$ utilizes a collimated $\mathrm{x}$-ray beam to illuminate the test article at a specific location while detectors mounted around the source measure photons scattered back from the test article, primarily via Compton scattering. This approach allows for a single sided radiographic type inspection. It also performs well on materials with low atomic numbers, such as SOFI, because the amount of Compton scattering is a function of the atomic number. Figure 2 shows a diagram and picture of the backscatter $\mathrm{x}$-ray system. 

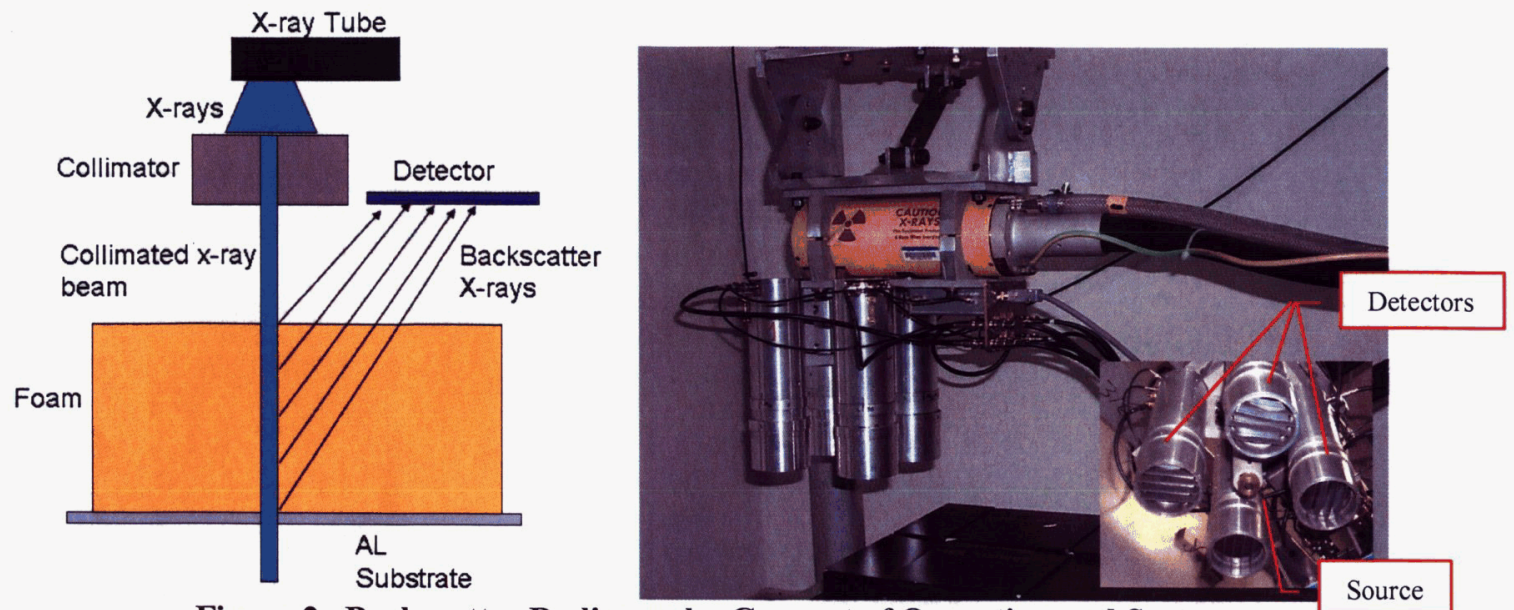

Figure 2. Backscatter Radiography Concept of Operation and System

Pulsed terahertz imaging works in a manner similar to the microwave method except that it operates at higher frequencies. For this application the center frequency point for $\mathrm{THZ}$ imaging was around $150 \mathrm{GHz}$, where microwave operated below $50 \mathrm{GHz}$. The higher frequency corresponds to a shorter wavelength, which increases the theoretical maximum resolution to enable detection of smaller defects than are discernable with the microwave techniques. At higher frequencies the difference between the dielectric constant of the SOFI and air becomes greater, allowing for more contrast in the image. The other difference is that this approach generates a pulse with broad frequency content instead of a single frequency at a time as the continuous wave microwave system does. This approach reveals more information about the foam properties and allows for more sophisticated processing techniques. Figure 3 shows a diagram and picture of the terahertz imaging system.

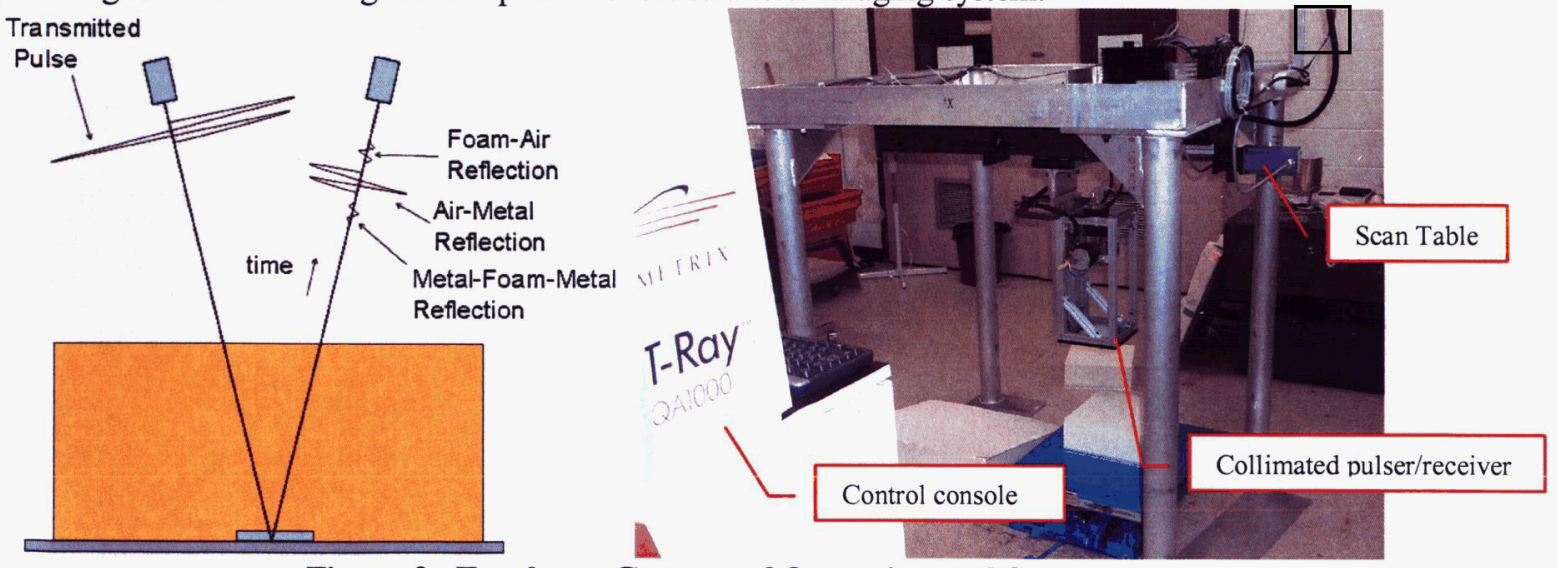

Figure 3. Terahertz Concept of Operation and System

A series of SOFI samples with artificial defects were sent to each of the vendors to build their experience working with foam and understanding how to interpret the data. After the development period was completed, each vendor received a sample in which they weren't told the location of the defects. Following the dissection of each test panel to verify the location of the planned and unintentional defects, the methods were graded based on how many of the defects they found, how many false calls they had and their technology readiness level. With this grading method terahertz imaging, microwave imaging and backscatter x-ray were ranked the highest. The other two methods yielded to high a false call rate and to low a detection rate to be useful for the types of defects given. Since the terahertz imaging method outperformed the microwave imaging method and both were similar in approach, the microwave method was dropped in favor of keeping backscatter x-ray which complemented the terahertz method. Backscatter is more sensitive to flaws near the surface because more photons scatter back from the foam near the surface than from the foam many inches below it. Also, because it does not rely on a reflection from the substrate, it is more effective over complex geometries where it is not possible to be normal to all tank features. The terahertz pulse is focused on the substrate so defects near the substrate are more visible than defects near the surface, which are outside the optimal depth of focus. 
In addition to the full development on these two methods, microwave imaging was also carried along at a lower level of effort as a backup in case terahertz failed to perform as expected or if further development allowed it to surpassed terahertz or backscatter. Shearography was worked just enough to keep it alive because of its potential for faster coverage and different defect detection capability. Even though shearography didn't do well for thick foam over complex substrates it did show promise for detecting crushed foam and delaminations in thin foam.

\section{Probability of Detection Test on Terahertz and Backscatter Systems}

Once the selection was made, contracts were initiated with the vendors to build "factory floor" hardened models and incorporate some advances made since the systems used for the down selection were built. When these models were received, a short set of experiments was performed to optimize the settings and then a Probability of Detection (POD) test was run. Based on the then current understanding of foam failure, artificial defect creation and foam inspection, the decision was made to use thin walled cylinders of cured foam for voids and thin sheets of cured foam for delaminations. Testing by way of cross sectioning through the sprayed over defect inserts showed that the SOFI inserts would blend in with the SOFI sprayed over them leaving a void for the hollowed out cylindrical inserts or a delamination under the thin wafer insert. These inserts were either glued to the substrate or inserted between spray passes depending on the desired height of the defect. Figure 4 shows samples of these defect inserts.
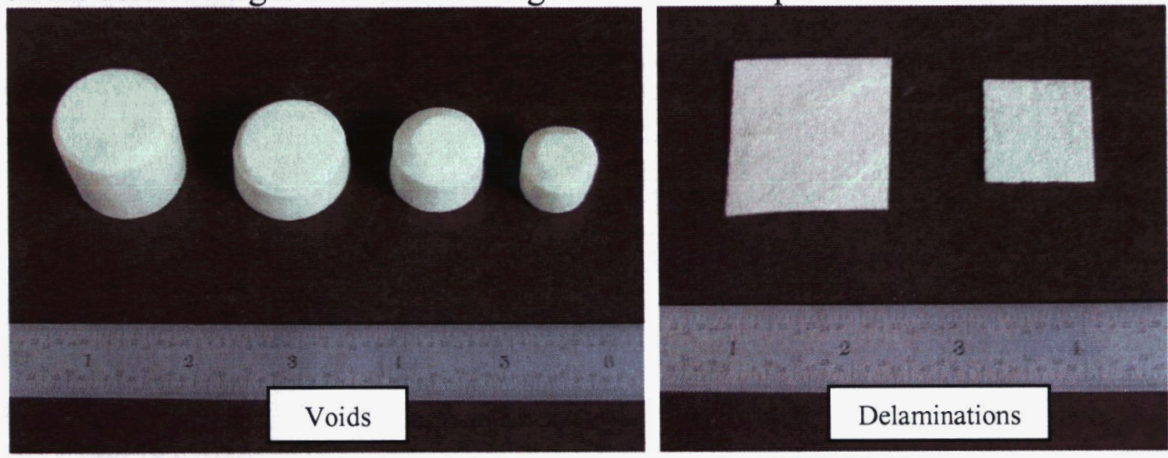

Figure 4. Artificial Defects Used for POD Test

The POD was designed using a 29 out of 29 statistical logic to obtain the required $90 \%$ detection capability with $95 \%$ confidence for a single defect size. The test was run with voids of three different diameters, starting at the targeted 1" diameter cylinder then going smaller in two steps down to a 0.5 " cylinder. Delaminations were not considered to be critical, but to determine basic NDE capability they were inserted in the test panels to provide a feel for their detection limits. Based on preliminary development testing it was determined that there was an effect of substrate geometry on detection capability. Certain geometries could mask a defect. To cover all the geometric variations, each of these defect sizes was placed in one of nine different locations. These areas, shown in Figure 5, were on and around the intertank stiffeners, on and around the flange and out in the flat acreage area of the tank. In addition to the previously mentioned variables, all the testing was run with two different foam thicknesses to determine detectability in both thick and thin foam simulating the PAL ramp and the hydrogen tank to intertank flange. With the exception of a small subset to test depth dependence, all defects were placed directly on the substrate. In order to accommodate all these variables, nearly 2000 defects were placed on 75 test panels. To gain an understanding of inspection repeatability and the dependence of results on the interpreter, the POD inspections were performed by four operators for each method and the data was analyzed by four different interpreters for each method.

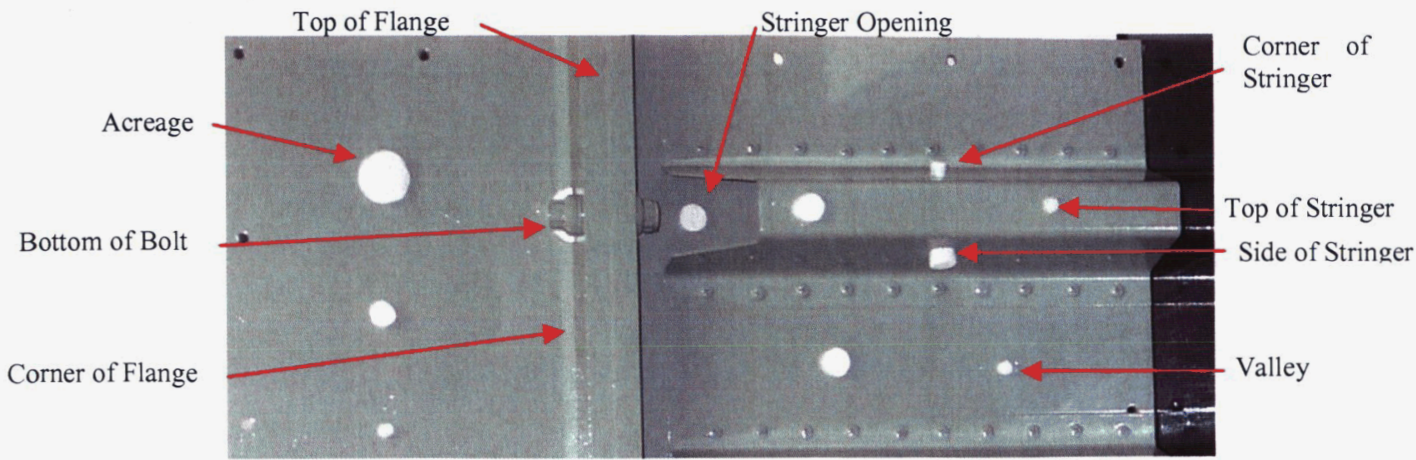

Figure 5. POD Panel Before Spray with Artificial Defects Applied 
The results of the POD test showed that terahertz imaging and backscatter x-ray methods had roughly equal success in detecting the inserted defects, and were able to find between $90 \%$ and $100 \%$ of the larger voids in simple geometries. Detection in complex geometries such as the corner of the flange and the sides and corner of the stringer was less successful and detection of delaminations was poor in almost every geometric location. Smaller voids were slightly more reliably detected with terahertz in thick foam and with backscatter in thin foam, as expected. Each panel was dissected to verify the final placement of all the SOFI defect inserts. During the dissection numerous natural voids, mostly very small, were identified. Detection of these natural defects was generally poor, and clearly did not reflect the trends observed in the POD. This revealed the difficulty in accurately determining the capability of the selected NDE methods to find defects that could be expected to occur on an ET. The approach of empirically determining a knockdown factor to relate the detection of an artificial defect to the detection of a natural defect ${ }^{4}$ has not been successfully applied because detection of this type of artificial defect has not been able to be correlated to detection of natural defects. The complex shapes and variable orientation of defects, in combination with the natural inhomogeneity of foam have made any sort of mathematical relationship elusive.

As a result of the POD work two studies were initiated to further define the capability of the two NDE methods. First, a study into detection of naturally occurring voids was initiated. Here, panels were sprayed with the intent of creating voids. The panels were then inspected with both methods and then dissected to determine the true defect count. The results showed that the threshold for detection was around the 1" level. The other factor which came out of the POD test was that the methods had a threshold of detection based on defect height. In order to quantify what that height was a series of test blocks with progressively shallower flat bottom holes were inspected. These blocks were stacked so that the effect of void depth versus height and diameter could be determined. As expected, the results showed that as defect height decreased so did signal strength. The detection threshold for a 1" diameter milled defect was found to be approximately 0.2". This confirmed the POD results that delaminations would be very difficult to detect with either method. A sample showing this trend for 1.0" voids under various levels of SOFI is given in Figure 6 .

\section{0" Milled Void}

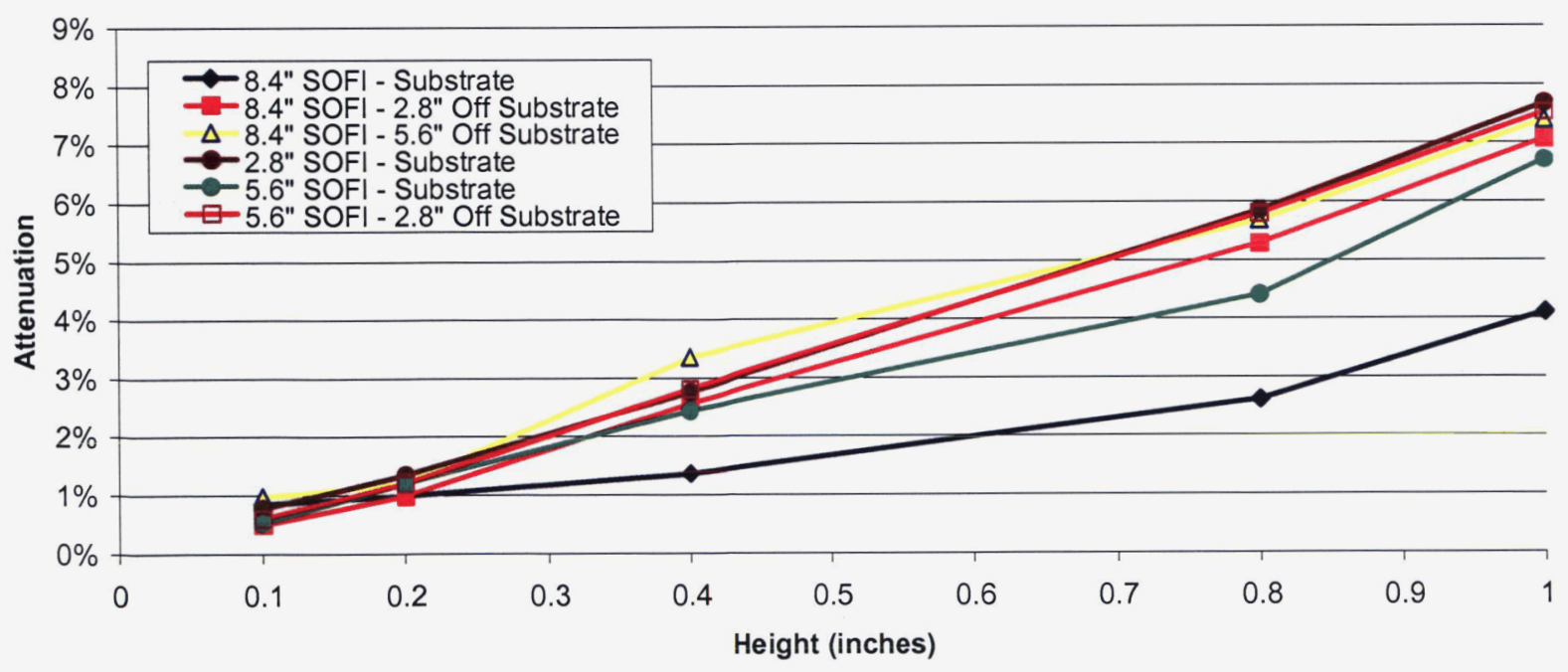

Figure 6. The Effect of Void Height on Defect Detection (Backscatter Radiography)

\section{Application to the External Tank}

By the time the POD study was completed a new understanding of the critical defect size had been reached and the defects of interest were determined to be much thinner than that used during the POD tests. This along with other complications kept the methods from being certified for SOFI acceptance testing but based on the experience obtained through this work, the decision was made to use backscatter radiography and terahertz imaging for engineering evaluation. That is, the methods could be used to look for defects, and those indications would be dealt with by an engineering review board, but the methods could not by themselves certify the quality of the SOFI. The ET Project Office directed that NDE be performed on the PAL ramps of the RTF tanks. The PAL ramps were the 
largest hand-sprayed close-outs and due to their simple substrate geometry, they had a good anticipated defect detection capability. Based on this direction, the PAL ramps on three ET's were inspected in preparations for RTF and by the time the decision was made to redesign the ET eliminating the PAL ramps a total of six ET PAL ramp sets had been evaluated with terahertz imaging and backscatter radiography.

During the time the POD study was conducted, tooling was fabricated to suspend the backscatter x-ray and terahertz imaging systems over the ET. The tooling made use of an overhead crane dolly to provide course axial and lateral motion over the tank, positioning the NDE scanning tables over the region of interest. A truss was mounted to the crane to get the scanning table to a fixed nominal height away from the tank. A three legged jack table was mounted from the truss that allowed the scanning table to be gimbaled. The jack table could then raise, lower and tilt the scan table to position the systems in the correct plane.

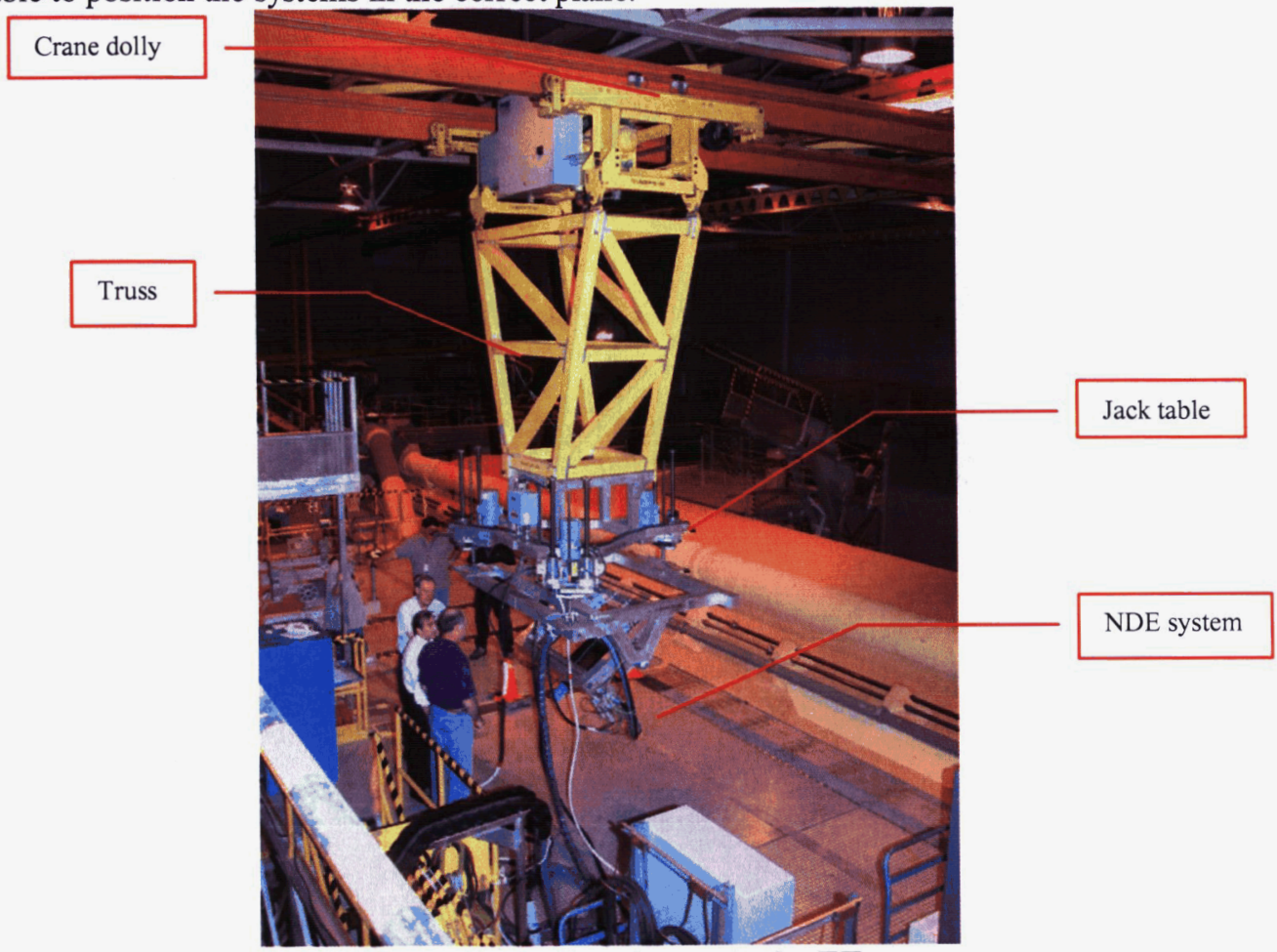

Figure 7. Tooling Access to the ET

The general procedure for inspecting the PAL ramps involved first marking a grid on the PAL ramps then methodically following that grid down the tank with each NDE system. A sample zone is shown in Figure 8 along with the accompanying backscatter $\mathrm{x}$-ray image.
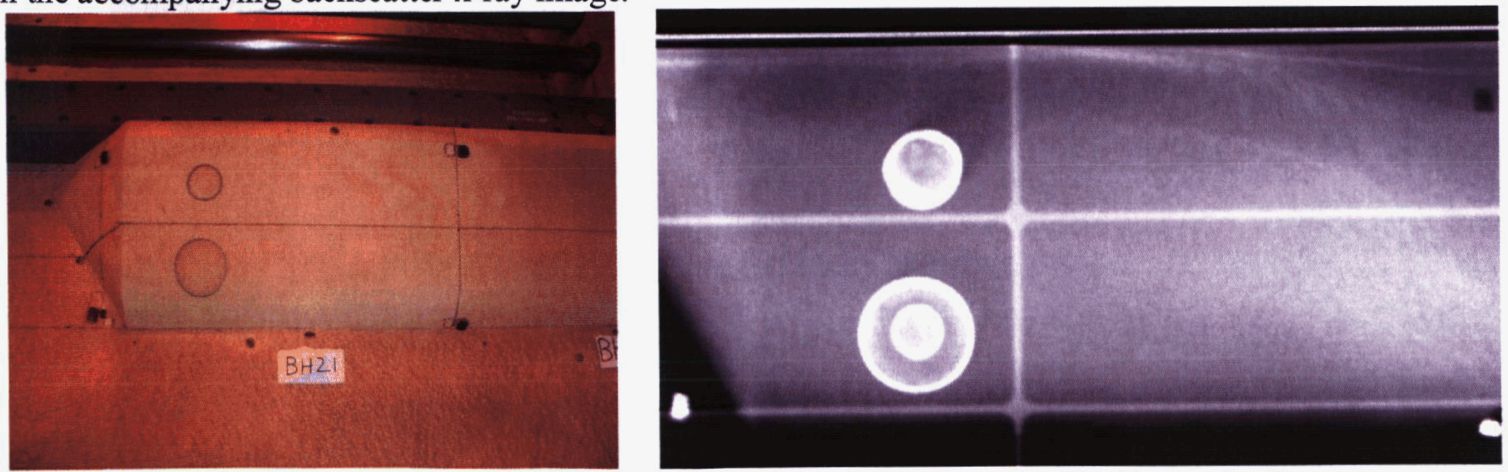

Figure 8. Typical Scan Zone and an Example Backscatter Radiography Scan

\section{Return to Flight and Beyond}

The flight of STS-114 was a success in many regards, but showed that there were still gaps in NASA's understanding of SOFI. During the launch of STS-114, the first Space Shuttle flight since the Columbia accident, a 
large piece of the foam broke off the PAL ramp and passed harmlessly under the Orbiter Discovery's wing. Based on the debris shape and the preflight NDE from the foam loss location it was determined that a void probably did not cause the debris. The new mechanism at work was most likely cracking and delaminating during ET fueling operations caused by the coefficient of thermal expansion mismatch between foam and the aluminum skin of the tank. These cracks formed a thermal short allowing air to liquefy near the skin of the tank and expand as it heated during ascent, providing a substantial increase in pressure to an already structurally compromised location. Considerable analysis and testing was performed on thermally cycled foam to characterize the nature of the crack growth.

This foam loss location was also the site of impact damage which was repaired while at the factory with a standard sand and blend procedure to remove the compromised foam. This raised the possibility that crushed foam may lead to foam loss and that the repair procedure was not fully effective. Because of this concern, a large number of tests were run on crushed foam to determine if it causes foam loss. The results of these tests showed that is can lead to small losses under some circumstances, but generally does not cause large scale foam loss like that observed on the PAL ramp.

With the new failure mechanism and critical defect type a fresh look at the types of NDE required was initiated. From this work shearography was brought back into play and was developed for crush damage and shallow delamination detection. This work and the shift from the PAL ramps to the IFR's during RTF-2, the time following STS-114 to STS-121 will be discussed in a follow on paper.

\section{References}

${ }^{1}$ Gehman, H. W., et. al., Columbia Accident Investigation Board: Report Volume I, Government Printing Office, Washington, D. C., 2003, Chapter 2.

${ }^{2}$ Kharkovsky, S., Hepburn, F., Walker, J., and Zoughi, R., "lisspection Of The Space Shuttle External Tank Sofi Using NearField And Focused Millimeter Wave Nondestructive Testing Techniques," Materials Evaluation, May 2005, p. 516.

${ }^{3}$ Dugan, E., Jacobs, A., Shedlock D., and Ekdahl, D., "Detection of Defects in Foam Thermal Insulation Using Lateral Migration Backscatter X-ray Radiography," Proceedings of SPIE $49^{\text {th }}$. Annual Meeting: Symposium on Optical Science and Technology, Penetrating Radiation Systems And Applications $V T$, Vol. 5541, Denver, August, 2004.

${ }^{4}$ Rummel, W., "Low Cost Method of Significantly Improving the Reliability of Instrumented NDT Procedures," 47th Annual NDT Forum, Air Transport Association of America, Inc., 2004. 